\title{
Smart sensor motion detection schemes in a noisy environment
}

\author{
Gregory P. Harmer and Derek Abbott \\ Centre for Biomedical Engineering (CBME) and \\ Department of Electrical and Electronic Engineering, \\ Adelaide University, 5005, South Australia
}

\begin{abstract}
Several motion detection schemes are considered and their responses to noisy signals investigated. The detection schemes include the Reichardt correlation detector, shunting inhibition neuron and the Horridge template model. These schemes are directionally selective and independent to the change in contrast. They essentially function by using spatial information and comparing it at successive time intervals. Using the detectors, the phenomenon of stochastic resonance (SR) is employed. SR is characterised by an improvement in response to a nonlinear system when noise is added to the input signal. Two types of SR are also considered, namely, subthreshold aperiodic and suprathreshold. Using stochastic resonance, the schemes are subjected to signals in an effort to improve the detectors responses with the addition of noise. We found that although added noise only further degrades the detectors response, an improvement can be gained by using some of the techniques from suprathreshold stochastic resonance.
\end{abstract}

Keywords: Smart sensors for motion detection, collision avoidance, insect vision, stochastic resonance, noisy sensory neural models

\section{INTRODUCTION}

In poor weather conditions millimetre waves offer a much greater penetration over the visible spectrum through small dust particles (aerosols), rain and fog. Antenna arrays capable of detecting mm-waves are able to be constructed. ${ }^{1}$ This design utilizes radiometry, which is the science of using passive detection techniques to detect background radiation. Unlike radar, which transmits a signal then receives the backscattered radiation, a radiometer only receives naturally occurring blackbody radiation. It is reasonable to expect then, in this passive detection system the signals are inherently noisy. Thus, the noise must be taken into account when processing the antenna array signals for the desired application.

The primary application is for a collision avoidance sensor, that is, a motion detector. This has stemmed from earlier work that developed a single 'seeing chip'. ${ }^{2-4}$ This functioned in the visible spectrum and implemented a simple 'insect template model' to detect motion..$^{5-7}$ The aim is to extend this to the mm-wave spectrum and utilize the noise to develop a mm-wave collision avoidance sensor.

A common belief is that addition of noise to a system always degrades the quality of the response, however, by use of the phenomenon of stochastic resonance, this is not always true. Certain non-linear systems have shown there is an optimal non-zero noise intensity that can be added to a system to improve its response. ${ }^{8}$ Originally developed for periodic signals, stochastic resonance has been extended to systems with either sub or supra-threshold broadband (aperiodic) signals. ${ }^{9}$

Three motion detection schemes have been investigated and we have evaluated the effects of stochastic resonance in the presence of noisy signals. The first is the Horridge template model, which is based on the mechanism the bee uses navigate. ${ }^{10}$ This is included for historical reasons, and also because of its simplicity to implement. The second is the Reichardt (or correlation) detector, which was the earliest explicit model in motion processing. ${ }^{11}$ The last involves shunting inhibitory neurons, ${ }^{12}$ which originated from a neurophysiological model. ${ }^{13}$

Results and conclusions detail the simulations used, and their success.

Correspondence: G. P. Harmer (gpharmer@eleceng.adelaide.edu.au) and D. Abbott (dabbott@eleceng.adelaide.edu.au). 


\section{MOTION DETECTION SCHEMES}

In order for a scheme to detect motion in a directionally selective way, certain minimum requirements must be satisfied; asymmetry, two inputs and a nonlinear interaction. ${ }^{14}$ Two inputs are necessary since motion is a vector, a single receptor could not distinguish a change in intensity as coming from either the left or right. A nonlinear interaction between the inputs is required, otherwise all the information about the temporal sequence is lost. This inhibits the sensor from being directionally sensitive. Finally, there must be some asymmetry between the two inputs, otherwise the input receptors could be switched without affecting the output, giving no directional selectivity.

There are several broad categories of detection schemes, which stem from the basis of their conception. Biological schemes based on cellular mechanisms or neurophysiology can be divided into gradient and correlation type of models. Gradient schemes estimate motion by relating the changes in spatial and temporal intensity, whilst correlation schemes are essentially based on the common delay and compare systems. The other broad category are the artificial schemes that take a more engineering type of approach.

Before going into details about the motion detection schemes, some criteria is established to determine the requirements of the schemes. ${ }^{12}$

- The sign of the response must indicate the direction of motion. This should be independent to the change in contrast of the moving object.

- There should be no response to a stationary edge or a varying contrast.

- Ideally it should be robust to noise.

- For an array of sensors, i.e. in the widefield, the position of the response should correspond to the moving edge. That is, spatially separated moving edges should each have a corresponding response.

The detectors that are considered in this paper are the Reichardt correlation detector, shunting inhibitory neuron and the Horridge template model.

\subsection{Reichardt Detector}

Also called the correlation detection, it is one of the earliest biological motion detection systems, based on the optomotor response of insects. ${ }^{11}$ The Reichardt elementary motion detection (EMD) detects motion in one direction by comparing the signal from one receptor to a delayed signal from an adjacent receptor. The comparison unit employs a simple multiplication, or correlation of the two signals. Due to the asymmetry of the EMD there exists a preferred and a null direction. That is, the response to a stimulus moving in one direction has a larger magnitude (preferred direction), to the response of the same stimulus moving in the opposite direction (null direction).

By combining two EMDs tuned to opposite directions, as shown in Fig. 1a, a bidirectional motion detector is produced. An important characteristic of the EMD is that it uses an excitatory mechanism to produce the response, i.e. the multiplicative interaction between the two channels.

The delay stage, represented by $\tau$, can be modeled as an exponential decay, which is the same as a first order low pass filter that has the transfer function $H(s)=1 /(s+A)$, where $A$ is the time constant. This allows better integration of delay stage into the biological modeling.

The response to a step input is shown in Fig. 1b. Consider a constant background of $L$ with a step of $(1+c) L$ moving from left to right, where $c$ is the contrast $(-1<c<1)$ and $L$ is the luminance. When the step reaches $R_{1}$, a larger response is produced on the negative side of the summer, thus giving a net negative response. This decays back to zero until the step reaches $R_{2}$. When the step reaches $R_{2}$, a larger response is produced on the positive side of the summer, giving a net positive response, which also eventually decays to zero.

This response does not appear to meet the criteria for a motion detector, however, by summing the responses from a number of adjacent Reichardt detectors (shown in Fig. 2a), the positive and negative responses almost cancel. In Fig. $2 \mathrm{~b}$ for example, the positive peak on the right would almost cancel with the negative peak on the left of the successive detector. This summing of adjacent detectors essentially creates a detection, thus the detection resolution is limited to that window. Since the positive and negative peaks have different magnitudes a small residue peak remains, the magnitude of which indicates the direction of motion. This is shown in Fig. $2 \mathrm{~b}$. The drawback with 
(a)

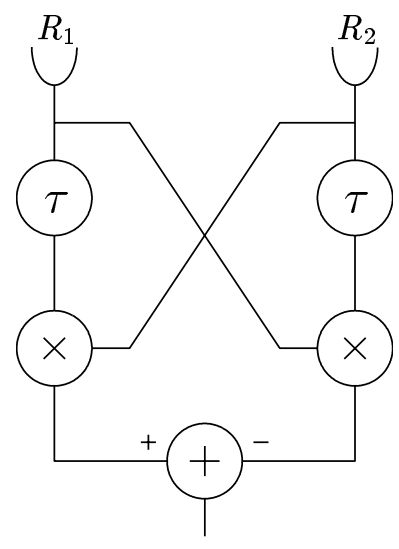

(b)

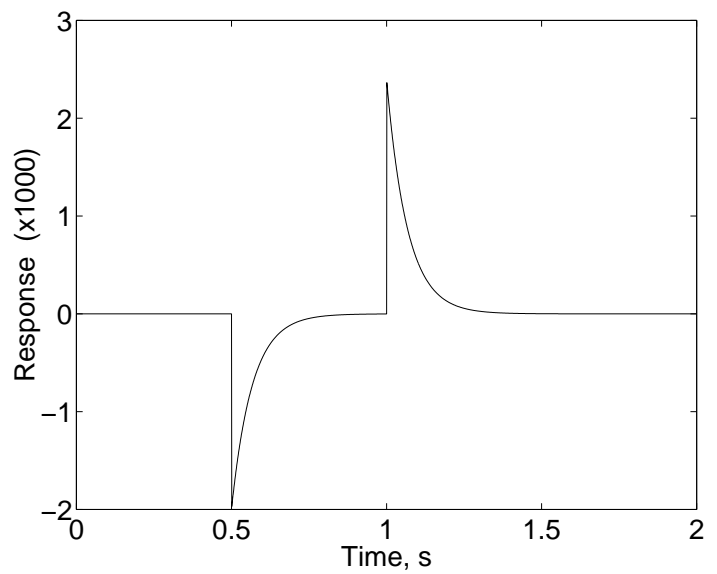

Figure 1. (a) Reichardt motion detector comprising of two EMDs, $\tau$ is the delay stage. (b) The response to a positive step input moving from left to right, $A=15$.

this technique is the large spikes each side of the detection window, the more EMDs per detection window the smaller their influence, but the poorer the resolution. The small 'bumps' within the detection window caused by the individual Reichardt detectors can be smoothed with the use of an additional low pass filter.

\subsection{Inhibitory Detector}

Rather than using an excitatory mechanism to compare channels, an inhibitory mechanism is also capable of providing a directionally selective sensor. The inhibitory mechanism proposed by Barlow \& Levick was a digital system derived from studies of directionally selective units in rabbit's retina. ${ }^{13}$ An alternative lateral inhibition mechanism, which resulted from studies of horseshoe crabs contains a linear interaction. ${ }^{15}$ A nonlinear version of lateral inhibition, called shunting inhibition was developed by Pinter by considering the neurochemistry of visual cells. ${ }^{16}$ Using this type of neuron, motion detection systems have been developed that have similar behaviour properties as the Reichardt detector. ${ }^{17}$ The shunting inhibition neuron is described by the following nonlinear equation,

$$
\frac{d m}{d t}=L-a m-m \sum_{i} k_{i} f_{i}\left(X_{i}\right),
$$

(b)

(a)

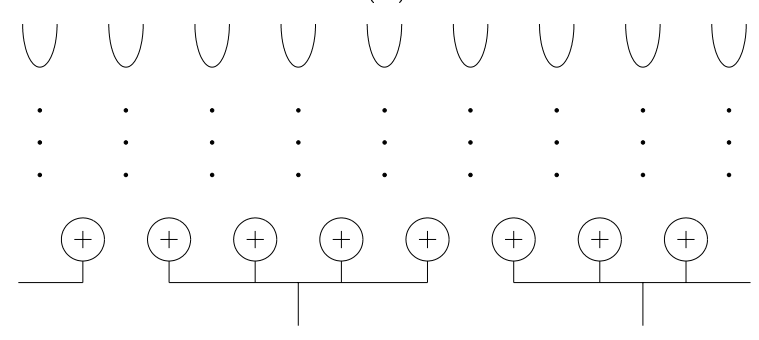

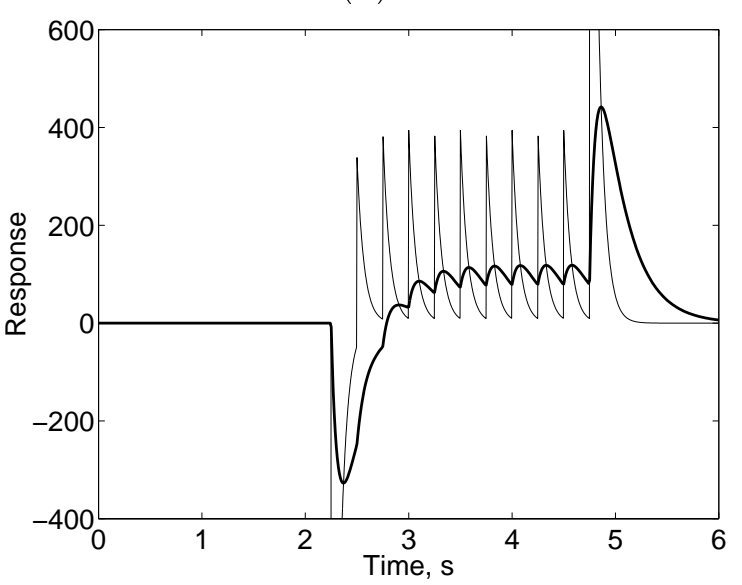

Figure 2. (a) The Reichardt detectors in the widefield configuration. (b) The thin lines show the response for one detection window consisting of 10 detectors $(A=15)$, the pulses from the individual detectors are quite visible. The thick line shows the low pass filtered $(A=4)$ output of the detection window. 
where $m(t)$ is the response (membrane voltage), $L(t)$ is the excitatory input, $a$ is the self decay of the neuron, $k_{i}$ are the weights, $f_{i}$ are the activation functions and $X_{i}(t)$ are the inhibitory inputs. With respect to diagrams of the neuron, a dot $(\bullet)$ represents the excitatory and a dash $(\boldsymbol{\omega})$ represents the inhibitory inputs, see Fig. 3 for example.

The modeling of the inhibitory neuron can be simplified by ignoring the internal dynamics, that is, setting $\dot{m}=0$. This is equivalent to the steady-state operation of the neuron and treats the neuron as a division operator giving

$$
m=\frac{L}{a+\sum k f(X)} \text {. }
$$

This simplifies the case studies when designing a detector based on this neuron, but in practice it is highly susceptible to noise and will not be used.

Similarly to the Reichardt detector, a local inhibitory motion detector can be configured, but it lacks the preferred and null direction characteristics. This can be overcome by constructing the inhibitory neuron network as shown in Fig. 3a. The difference is that the excitatory input to both neurons is provided by the same receptor. The inhibitory inputs are provided by the other receptor, one being delayed for the asymmetry. A consequence of this is that the response is zero until the stimulus reaches the inhibitory input. This is useful, as we shall see shortly when creating the bidirectional motion sensor.

(a)

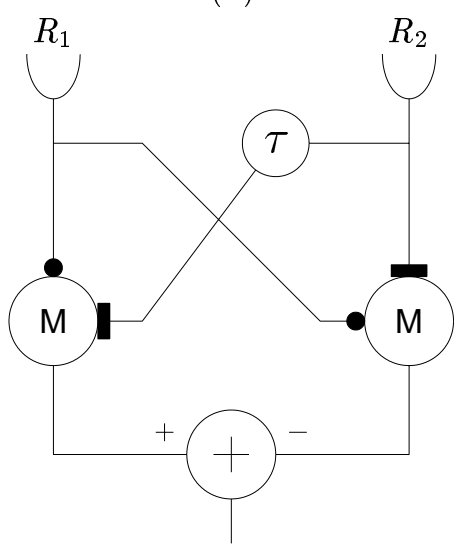

(b)

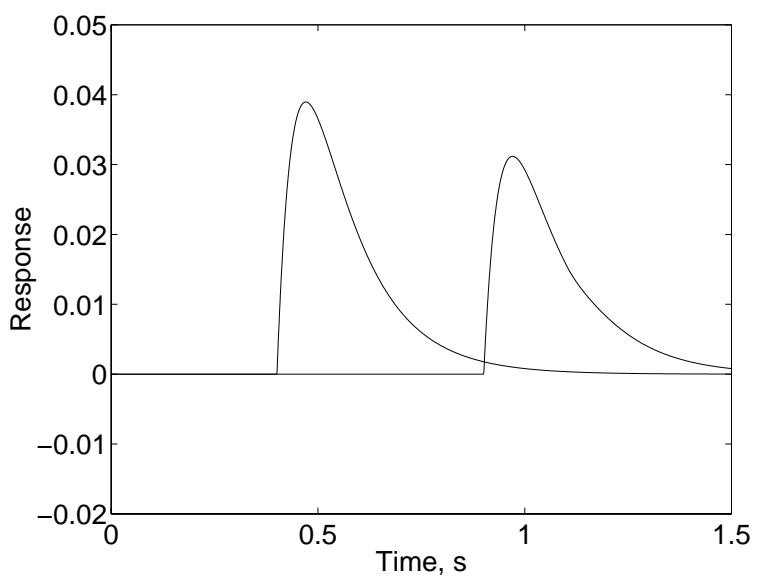

Figure 3. (a) Local inhibitory detector, $M$ is the shunting inhibitory neuron. (b) The right peak is the response for a positive step input moving from the left to the right, while the left peak is due to the same edge moving from the right to the left. This is the preferred and null direction respectively. $(a=10, A=8, c=0.25, L=10)$

However, the arrangement of Fig. 3a has two problems, similar to those of the EMD. Firstly, the sign of the response is dependent on the change of contrast, this is easily seen by considering a step input in the steady-state. Secondly, the peaks to the same step input moving in opposite directions give responses of different magnitudes. There is a preferred and a null direction, ${ }^{17}$ ideally the magnitude would give some indication of the edge properties.

Though this arrangement needs modifying, the same trick as in the Reichardt detector can be employed, namely placing two detectors back-to-back. ${ }^{12}$ This forms the directionally sensitive local inhibitory motion detector (DSLIMD), as shown in the widefield configuration in Fig. 4a. The detector response is given by $y_{i}$ as shown in Fig. 4a. This response depends on the receptors $R_{i-1}, R_{i}$ and $R_{i+1}$, but since no response is generated to either of the inputs of the summer $y_{i}$ until the stimulus reaches $R_{i}$, a symmetrical sensor results. The response $y_{i}$ is also now independent to the change of contrast of the stimulus.

The response to a step input are shown in Fig. 4b, which is simply the difference of the two curves shown in Fig. 3b, taken at appropriate times. The steady state response is also shown on the same plot. The response the DSLIMD gives is superior to the Reichardt detector as it does not require averaging over the window of detectors, and it does not have the unpleasant spikes either side of the detection window.

To preliminary investigate the noise resilience of the DSLIMD with and without the internal dynamics, both systems were subjected to the same noisy signal. The results shown in Fig. 5 clearly show that the internal dynamics make a difference to the clarity of the response. 
(a)

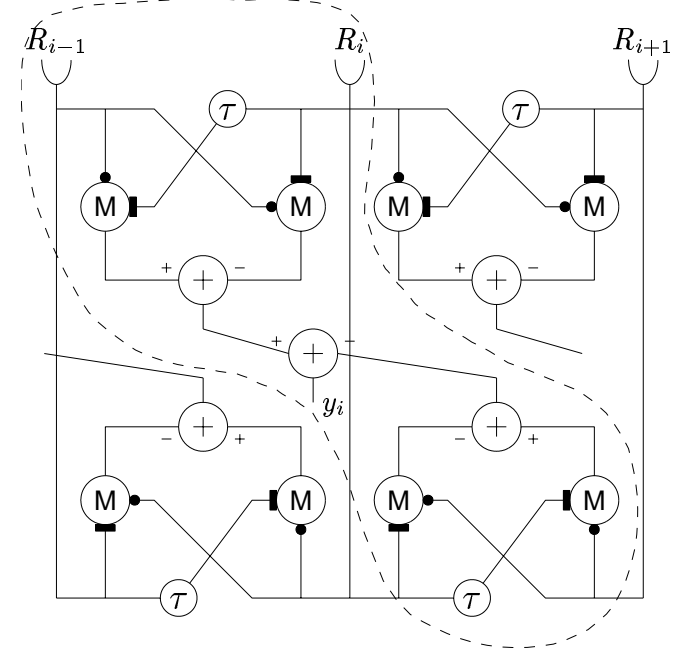

(b)

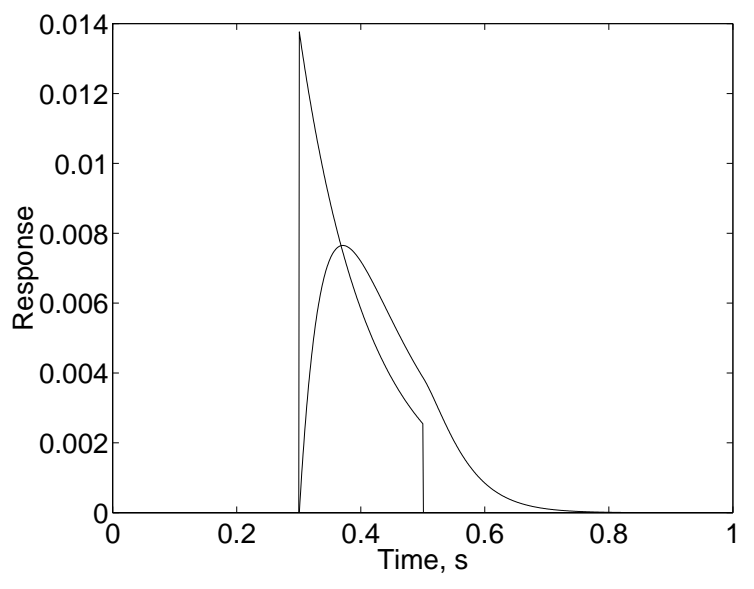

Figure 4. DSLIMD and response. (a) The dashed outline shows a single slice of the DSLIMD from the widefield configuration. In practice only one time delay element is required per receptor, two are drawn here for clarity. (b) The response to a positive step input moving from the left to the right, parameters as for Fig. 3. The curved trace utilizes the internal dynamics of the neuron while the other trace is the steady state response.

(a)

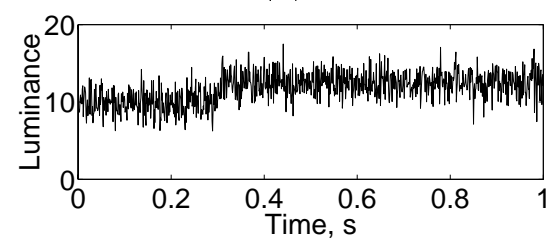

(b)

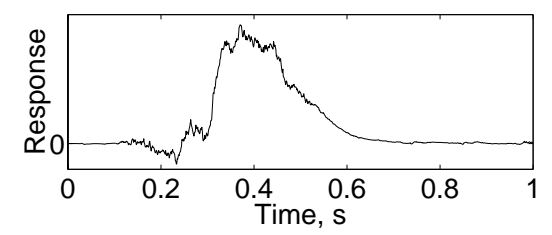

(c)

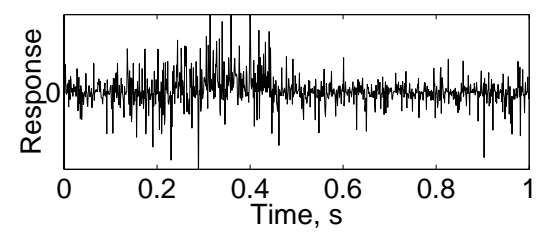

Figure 5. Rudimentary noise analysis of the DSLIMD detector. (a) The test stimulus. (b) Response of the DSLIMD using the full internal dynamics of the inhibitory neuron. (c) Response in the steady-state, which is greatly affected by noise.

\subsection{Template Model}

The template model, originally developed by Horridge to model insect vision, shares both biological and engineering heritage. ${ }^{10,18}$ A diagram of the template model is shown in Fig. 6a. The input channels are first bandpassed filtered (the same as differentiating with respect to time), which indicates the change in intensity. This is then quantised by thresholding to give one of three states, increase $(\uparrow)$, decrease $(\downarrow)$ or no change $(-)$ in intensity. Templates are then formed by considering adjacent spatial channel at successive time intervals. This forms a $2 \times 2$ template that contains both spatial and temporal information, which is then used with a look up table. Since there are three possibilities for each of the four quantised intensity changed values in the template, there are a total of 81 possible templates. By considering each of the templates, there are only eight that indicate coherent motion, which are known as directionally motion-selective templates (DMST). ${ }^{5}$ Although there are others that have the required 3:1 diagonal asymmetry that indicate motion, the eight DMSTs exercised in this paper are those most immune to noise. By tracking particular templates, quantities such as velocity and time to impact can be calculated. ${ }^{6,7}$

The response of the template model to a spot moving back and forth is shown in Fig. 6b. This differs from the previous two schemes as the template model tracks the leading and lagging parts of the edge, not the edge itself ${ }^{19}$ Therefore, for each edge two templates appear, as shown the response (Fig. 6b) where there are two edges. The other difference is it does not have any type of reliability measure. That is, for the previous schemes, the larger the magnitude of the response the higher the probability that there was motion. The template model has a discrete output, either there is motion (left or right) or there is none, no matter the contrast difference for example. 
(a)

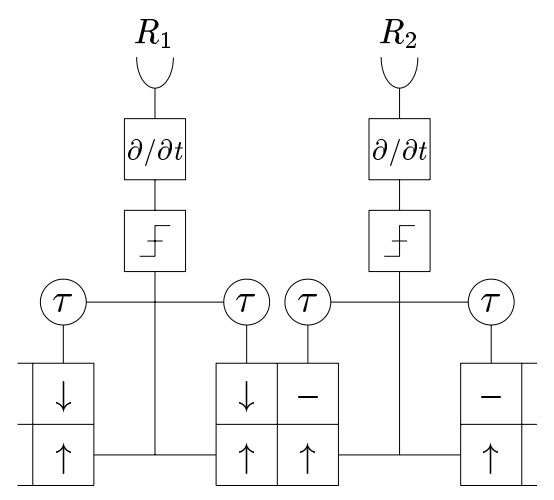

(b)

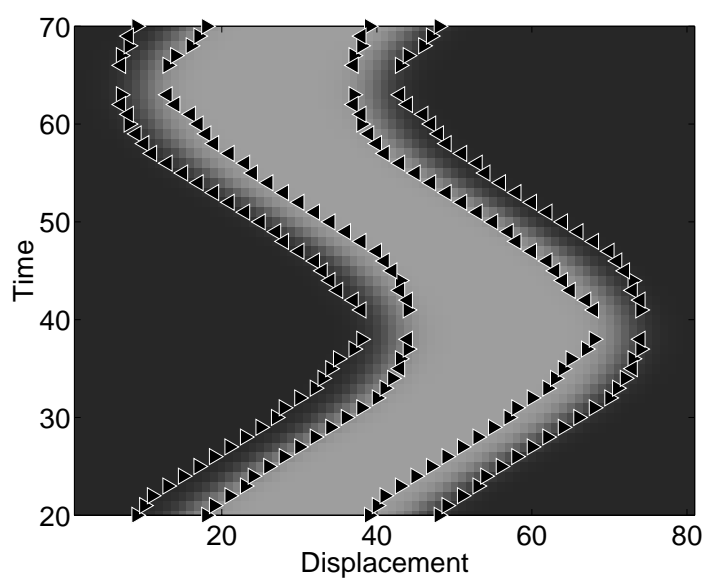

Figure 6. Template model and response. (a) A section of the widefield schematic of the template model. The signal from the receptors is differentiated, thresholded, delayed and stored as a $2 \times 2$ template. (b) The response to a step input being moved back and forth, $\triangleleft$ and $\triangleright$ denote leftward and rightward motion respectively.

\subsection{How to Quantify}

In communication systems, the 'goodness' of the detector can be easily be quantified since its output should match the source as closely as possible. However, the output of motion detection schemes does not resemble that of the source (images, for example), so some interspike steps are necessary. The intuitive action to take is to create a benchmark, or some type of template from a clear signal. Mathematically, we can predict where the motion, $M(x, t)$ is from the instantaneous spatial and temporal gradients as

$$
M(x, t)=-\frac{\partial L}{\partial x} \frac{\partial L}{\partial t}=-L_{x t},
$$

where $L$ is the luminance, $x$ and $t$ are space and time respectively. This provides a contrast independent measure of the motion, the minus sign orientates a positive $M$ as being rightward. Using this quantity as a benchmark, the performance of the motion detection schemes can be measured, via correlation or mean square error types of metric. It is important to use (3) only on clean images such as those generated by computer, due to differentiation operators being inherently noisy.

Although (3) is sufficient for the Reichardt and DSLIMD schemes, it is not useful for the template model. The template model detects leading and lagging parts of an edge, thus we require a benchmark such as,

$$
\hat{M}(x, t)=\operatorname{sign}(M)\left|L_{x x} L_{t t}\right| .
$$

In the first instance, simple normalised correlations or mean square error of the differences will be used. An information theoretic measure described in the next section would ideally be used if possible.

\section{STOCHASTIC RESONANCE}

There are large amounts of literature relating to stochastic resonance (SR) involving periodic signals (see Ref. 8 for an extensive review), thus we will limit this discussion to the newer types of SR. They are aperiodic stochastic resonance (ASR) and suprathreshold stochastic resonance (SSR).

The term ASR, first coined by Collins ${ }^{20,21}$ in 1995 describes a SR system that works with broadband signals. Clearly, the previous techniques using signal-to-noise ratio or modes in interspike interval histograms ${ }^{22}$ are not appropriate for ASR. Collins et al. proposed a new measure, the power norm, with the normalised version given as

$$
C_{1}=\frac{\overline{s(t) r(t)}}{\left[\overline{s(t)^{2}}\right]^{1 / 2}\left[\overline{(r(t)-\overline{r(t)})^{2}}\right]^{1 / 2}}
$$


where $s(t)$ is the zero mean aperiodic signal and $r(t)$ is the response of the system. The overbar denotes an average over time. Essentially this is a simple input-output cross-correlation, as determined by the numerator, which is taken at a time lag of zero. In general, we wish to utilize the measure that quantifies the peak in the correlation function. ${ }^{21}$ The two terms in the denominator are equivalent to the standard deviations of the signal and response, their purpose to normalise the correlation.

While ASR (and periodic SR) improve the response with the addition of noise, it lacks robustness due to the sensitive setting of the threshold. If the signal strength increases past the threshold the addition of noise only serves to decrease $C_{1}$, which removes the $\mathrm{SR}$ from the system. It is shown that a much improved response can be gained by lowering the threshold so an output is always produced, ${ }^{23}$ although this requires a different system architecture. This leads to the term SSR, where the signal is suprathreshold, with the threshold usually placed at the signal mean. This improves the robustness of the system, as the threshold setting is independent of the signal strength.

It has also been pointed out that one of the problems with using the measure $C_{1}$ is that it is a linear indicator based on linear response theory. ${ }^{24,25}$ Although linearisation occurs for large enough amplitudes of noise, ${ }^{26}$ the system is designed primarily to be nonlinear. So it seems inappropriate to use correlations, especially for small noise intensities. A more general indicator is the amount of information transmitted through a system, referred to as the average mutual information (AMI) ${ }^{9}$ In this setting we consider a network of $N$ devices, each with independent noise sources as shown in Fig. 7a.

(a)

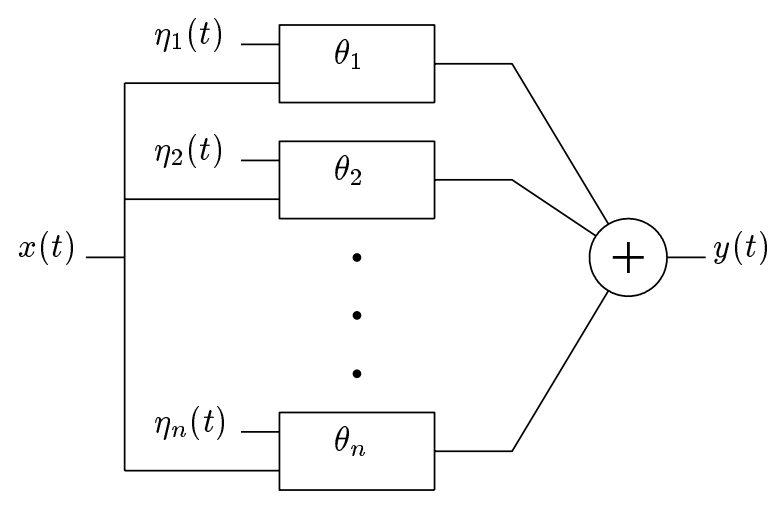

(b)

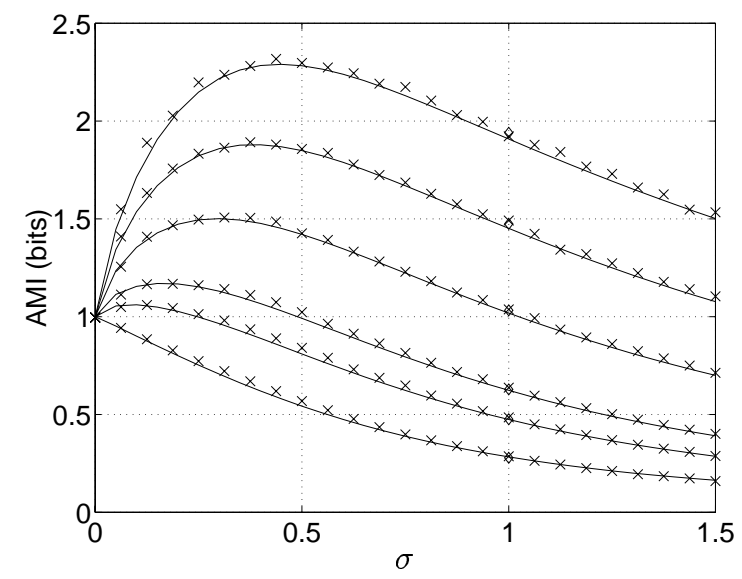

Figure 7. (a) A summing network of $N$ devices. (b) The AMI against the normalised noise $\sigma=\sigma_{\eta} / \sigma_{x}$ for various $N$ with all $\theta_{i}=0$, from bottom up the values of $N$ are 1,2,3,7, 15 and 31. The crosses are from simulations of the network and the lines are from numerically evaluating (7).

Using the most simple nonlinear devices, the Heaviside function as described by

$$
y(t)=\left\{\begin{array}{ll}
1 & \text { if } x(t)+\eta_{i}(t)>\theta_{i} \\
0 & \text { otherwise }
\end{array},\right.
$$

where $\eta_{i}(t)$ are the noise and $\theta_{i}$ are the thresholds, $x(t)$ and $y(t)$ are the input and output respectively. Thus, the response of the network is equal to the number of devices that have been triggered. Any model can be used in the device, usually some type of neuron model, but the Heaviside function is used for simplicity.

Having established the threshold network, the transmitted information is written as ${ }^{9}$

$$
\begin{aligned}
\mathrm{AMI} & =H(y)-H(y \mid x) \\
& =-\sum_{n=0}^{N} P_{y}(n) \log P_{y}(n)-\left(-\int_{-\infty}^{\infty} \sum_{n=0}^{\infty} P_{x}(x) P(n \mid x) \log P(n \mid x) d x\right)
\end{aligned}
$$

where $H(y)$ is the information content (or entropy) of $y(t)$ and $H(y \mid x)$ is the amount on information lost in the transmission. $P_{y}(n)$ is the probability the output $y(t)$ is $n$ and $P(n \mid x)$ is the conditional probability density of the 
output being $n$ given the signal value $x$. The logarithms are taken as base 2, thus the AMI is in bits. The AMI for several values of $N$ (number of devices) are shown in Fig. 7b, both from simulations of the system and numerically integrating (7). As mentioned earlier, an improvement due to SSR can be achieved for varying signal strength without modifying the threshold. This is true as long as the noise scales accordingly to signal strength, which is highlighted by the $x$-axis of Fig. 7b. This defines the noise intensity relative to the signal intensity as $\sigma=\sigma_{\eta} / \sigma_{x}$, where the $\sigma$ 's are the standard deviations of the respective signals.

It was pointed out by Stocks and colleagues that placing all the thresholds at the same value is an inefficient use of $N$ devices. Finding the set of optimal threshold, $\left\{\theta_{i}\right\}$, is a problem in "optimal quantisation" where one must maximise the AMI. ${ }^{27}$ Given a system has a priori knowledge of the input signal, one can find the optimal threshold settings. For a uniformly distributed signal, uniformly distributing the thresholds between the limits of the signal leads to optimal threshold levels. ${ }^{25}$ Note that the system is only optimal in the absence of noise, as the noise changes the distribution of the signal. The important result is that when noise is added to a system with optimal thresholds, it only proves detrimental to the system.

\section{SIMULATIONS}

Simulations were performed with two test sequences, the first consisted of a single spot moving back and forth, the second consisted of three spots all moving back and forth $\left(120^{\circ}\right.$ phase difference). Since the motion detectors only function along one spatial dimension, only a vector image sequence was used. Thus, for 2-dimensional processing all the horizontal lines are independent. Having only a vector image also simplifies the spatial and temporal calculations and displaying the results on the printed page.

Due to the large spikes at either edge of the detection window for the Reichardt detector, it was not considered for analysing. This could be minimized by adding a bandpass unit, but being an excitatory type of detector, the noise will adversely affect the performance of the detector in the same way as in the steady state inhibitory detector.

A network was set up as per the diagram in Fig. 7, with a DSLIMD as the device. Alternatively, one can think of the widefield configuration shown in Fig. 4a being in parallel with itself $N$ times. The responses of the DSLIMDs are added to give the response of the system. The correlation coefficient is calculated based on (5), but taking into account the extra spatial dimension. The input signal was generated using (3) from a noiseless image sequence. The delay between the input and output was compensated for in order to produce the maximal input-output correlation. The results of the DSLIMD to the test signals are shown in Fig. 8, the correlations in (a) and mean square errors (of the normalised signals) in (b).

It is clear from Fig. 8 that adding noise only leads to degrade the response. The correlations monotonically decrease with noise as well as the mean square error (MSE). A possible explanation to this intuitive result is that

(a)

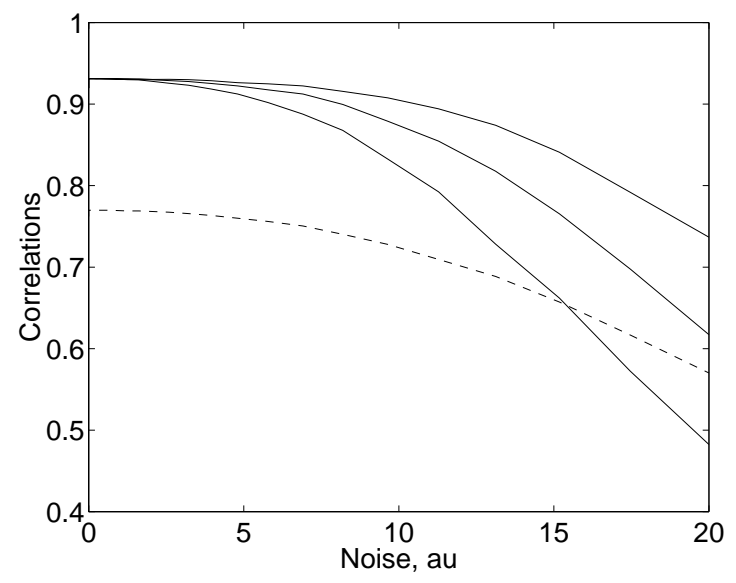

(b)

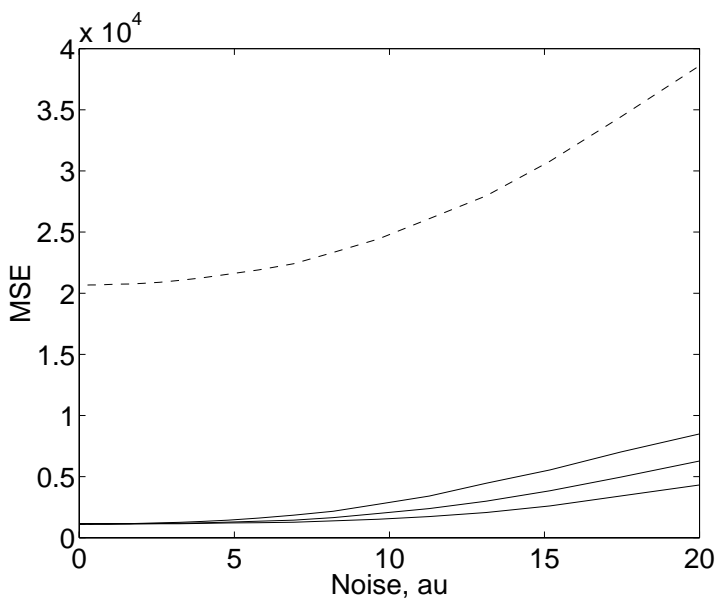

Figure 8. Results of the widefield DSLIMD. (a) The normalised correlations where the solid lines are for a single spot moving back and forth with $N=7,15$ and 31 from bottom to top and the dashed line is for three spots moving with $N=15$. (b) The mean square error (MSE) with the lines as for (a). 
the system may be optimal. Consider the SSR system with the devices as Heaviside functions with distributed thresholds as mentioned earlier. This system is also considered to be optimal, where the addition of noise similarly degrades the system performance. It is not until some compromise or constraint is made, that the addition of noise may be useful. Although noise does degrade the system performance, a positive result is the correlations increase for larger values of $N$. This is due to the summing (or averaging) of the responses and the Gaussian properties of the noise.

Due to the template model having separable functional units, there are several ways it can be configured in the network architecture. We have considered two configurations, having the whole model as the device or networking up to the threshold units. The first configuration is much more computationally intensive as the template model must be formed in every device whereas for the second, only one template model is formed. In Fig. 9, the fully and partially networked systems are denoted by the solid and dashed lines respectively.

Figure 9 shows the correlations for the template model for various values of $N$. The correlations were taken between the system output (motion vectors) and the signal $\hat{M}$ generated by (4). The thresholds were chosen to match the placement of the motion vectors as close as possible to the peaks in $\hat{M}$, thus maximising the correlation coefficient. Similarly to the DSLIMD system, the addition of noise only tends to degrade the performance of the system. Additional networked devices, similarly, improve the correlation. The correlation values found with zero noise intensity can be misleading as no normalisation has been used.

Comparatively, for the same noise intensity, the DSLIMD provides a superior response. This is clearly observable when viewing the inputs and outputs visually. This is due to the implicit noise immunity of the DSLIMD as shown in Fig. 5. The template model falters due to its 'abstract' processing of the templates, noise may alter only one intensity change value, but several templates may be adversely affected.

\section{CONCLUSION}

We have shown that with these motion detection schemes, in a simple network configuration, no benefit is gained by adding noise to the signal. A possible reason being that devices are functioning optimally, and additional noise only degrades an optimal system. However, with the sensors arranged in the network architecture, an improvement in the input-output correlation is gained as the number of devices increase.

In this work, quantitative results were achieved via the use of correlations, which were based on Collins et al. original power norm $C_{1}$. Further work is required in order to implement the information theoretic measure. It still remains unclear how this system could be transformed appropriately to apply the AMI metric.

Further investigations into different network architectures and modified detection schemes are also required.

(a)

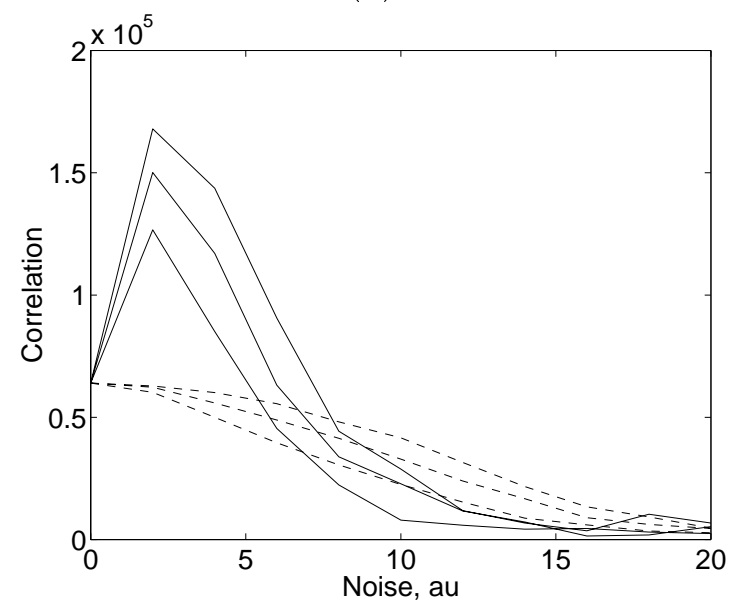

(b)

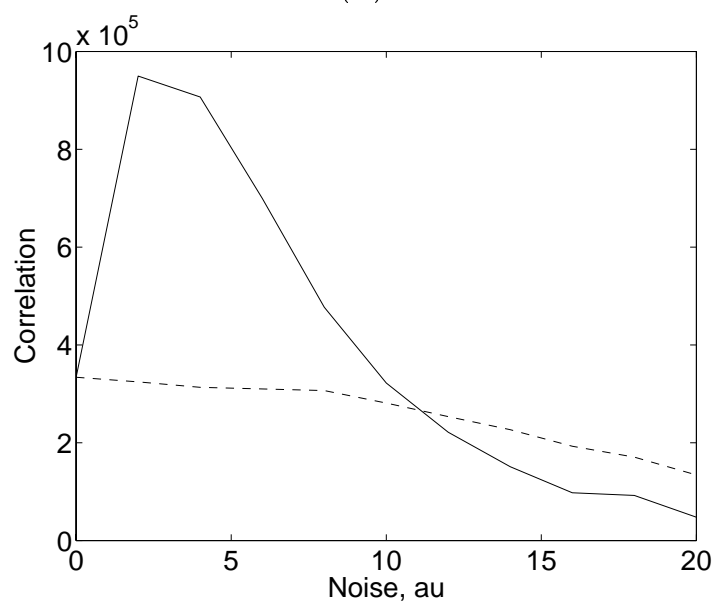

Figure 9. Results of the template model, $N=7,15$ and 31 from bottom to top. (a) Correlations for a single spot moving back and forth, two different configured systems are represented by the line styles are described in the text, a threshold of 8 was used. (b) Correlations for three moving spots, lines as in (a), $N=15$ and a threshold of 6 . 


\section{ACKNOWLEDGMENTS}

This work was funded by the Australian Research Council and the Sir Ross and Sir Keith Smith Fund.

\section{REFERENCES}

1. D. C. Goodfellow, G. P. Harmer, and D. Abbott, "Millimeter-wave collision avoidance sensors: future directions," in Mobile Robots XIII and Intelligent Transportation Systems, vol. 3525 of Proceedings of SPIE, pp. 352-362, 1998.

2. D. Abbott, A. Moini, A. Yakovleff, X. T. Nguyen, A. Blanksby, G. Kim, A. Bouzerdoum, R. E. Bogner, and K. Eshraghian, "A new VLSI smart sensor for collision avoidance inspired by insect vision," in Proceedings of SPIE, vol. 2344, pp. 105-115, (Boston, MA), 1994.

3. A. Moini, A. Bouzerdoum, A. Yakovleff, and K. Eshraghian, "A two dimensional motion detector based on the insect vision," in Advanced Focal Plane Arrays and Electronic Cameras, pp. 146-157, SPIE, (Berlin, Germany), 1996.

4. X. T. Nguyen, A. Yankovleff, A. Moini, K. Eshraghian, A. Bouzerdoum, and R. E. Bogner, "VLSI architecture of a low computational load processor for a visual system based upon insect vision," in Proceedings of the European Design 83 Test Conference, User Forum, pp. 85-89, (Paris, France), 1994.

5. X. T. Nguyen, A. Bouzerdoum, R. E. Bogner, A. Monin, and K. Eshraghian, "Feature representation of motion trajectories," in Proceedings of the IEEE International Conference of Neural Networks, vol. 6, pp. 2922-2927, 1995.

6. A. Yakovleff and A. Moini, "From sensing to perceiving: An overview of the bugeye project," in Advanced Focal Plane Arrays and Electronic Cameras, pp. 86-96, (Berlin, Germany), 1996.

7. R. Beare and A. Bouzerdoum, "Two-dimensional motion computation using a template model," in Proceedings of the International Conference on Systems, Analysis and Synthesis, pp. 849-55, (Orlando, Florida), 1996.

8. L. Gammaitoni, P. Hänggi, P. Jung, and F. Marchesoni, "Stochastic resonance," Reviews of Modern Physics 70, pp. 223-287, Jan. 1998.

9. N. G. Stocks, "Suprathreshold stochastic resonance in multilevel threshold systems," Physics Review Letters 84(11), pp. 2310-2313, 2000.

10. G. A. Horridge, "A template theory to relate visual processing to digital circuitry," Proceedings of the Royal Society of London B 239, pp. 17-33, 1990.

11. W. Reichardt, "Autocorrelation, a principle for the evaluation of sensory information by the central nervous system," in Sensory Communication, W. A. Rosenblith, ed., Contributions to the Symposium on principles of sensory communication, pp. 303-317, The MIT Press, (Endicott House, MIT), 1961.

12. R. Beare, Image segmentation based on local motion detection. PhD thesis, University of Adelaide, 1997.

13. H. B. Barlow and W. R. Levick, "The mechanism of directionally selective units in rabbit's retina," Journal of Physiology 178, pp. 477-504, 1965.

14. A. Borst and M. Egelhaaf, "Principles of visual motion detection," Trends in neurosciences 12(8), pp. 297-306, 1989.

15. H. K. Hartline and R. Ratliff, Studies in excitation and inhibition in the retina, Rockefeller University Press, New York, 1974.

16. R. B. Pinter., "The electrophysiological bases for linear and for nonlinear product term lateral inhibition and the consequences for wide field textured stimuli," Journal of Theoretical Biology 105, pp. 233-243, 1983.

17. A. Bouzerdoum, "The elementary movement detection mechanism in insect vision," Phil. Transactions of the Royal Society of Londan B 339, pp. 375-384, 1993.

18. G. A. Horridge and P. Sobey, "An artificial seeing system copying insect vision," International Journal of Optoelectronics 6(1/2), pp. 177-193, 1991.

19. X. T. Nguyen, Smart VLSI micro-sensors for velocity estimation inspired by insect vision. PhD thesis, University of Adelaide, 1996.

20. J. J. Collins, C. C. Chow, and T. T. Imhoff, "Stochastic resonance without tuning," Nature 376, pp. 236-238, 1995.

21. J. J. Collins, C. C. Chow, and T. T. Imhoff, "Aperiodic stochastic resonance in excitable systems," Physical Review E 52(4), pp. 3321-3324, 1995. 
22. A. R. Bulsara and L. Gammaitoni, "Tuning into noise," Physics Today 49(3), pp. 39-45, 1996.

23. D. R. Chialvo, A. Longtin, and J. Müller-Gerking, "Stochastic resonance in models of neuronal ensembles," Physical Review E 55(2), pp. 1798-1808, 1997.

24. N. G. Stocks and R. Mannella, "Suprathreshold stochastic resonance in neuronal network model," in In Press, 1999.

25. N. G. Stocks, "Suprathreshold stochastic resonance," in Stochastic and Chaotic Dynamics in the Lakes, D. S. Broomhead, E. Luchinskaya, P. McClintock, and T. Mullin, eds., vol. 502, pp. 415-421, American Institute of Physics, (Melville, NY, USA), 2000.

26. M. I. Dykman, D. G. Luchinsky, R. Mannella, P. V. E. McClintock, H. E. Short, N. D. Stein, and N. G. Stocks, "Noise-induced linearisation," Physics Letters A 193, pp. 61-66, 1994.

27. N. G. Stocks, "Suprathreshold stochastic resonance and optimal quantization," Submitted, 2000. 